\section{Autoavaliação da saúde em idosos: pesquisa de base populacional no Município de Campinas, São Paulo, Brasil}

\author{
Self-rated health in the elderly: a population-based \\ study in Campinas, São Paulo, Brazil
}

\begin{abstract}
This study analyzed self-rated health in the elderly according to demographic, socioeconomic, and health-related behavior. This was a cross-sectional, population-based study with a cluster sample using data from a survey in Campinas, São Paulo State, Brazil (ISACamp 2008-2009). Adjusted prevalence ratios were estimated using multiple Poisson regression. The study enrolled 1,432 elderly. Prevalence of excellent/very good health was 24.6\% and was significantly higher in the elderly with more schooling, higher income, no religion, living alone, home computer, alcohol consumption 1-4 times a month, physical activity during leisure-time, no obesity, and fruit and vegetable consumption $\geq 4$ times a week. Some of the findings are scarce in the literature and indicate relevant topics for further investigation. The results highlight the need for greater attention to socially vulnerable groups and the development of strategies to promote healthy habits in the elderly.
\end{abstract}

Health of the Elderly; Health Surveys; Self-assessment
Flávia Silva Arbex Borim 1

Marilisa Berti de Azevedo Barros 1

Anita Liberalesso Neri 1

\section{Introdução}

A autoavaliação da saúde tem sido amplamente utilizada como importante indicador de bemestar individual e coletivo $1,2,3$. É empregada também como robusto preditor de morbidade ${ }^{4}$, incapacidade 5 , depressão ${ }^{3}$, inatividade ${ }^{6}$ e mortalidade 7,8, especialmente entre idosos.

As autoavaliações de saúde são geralmente obtidas em inquéritos de saúde de base populacional por meio de uma pergunta simples, de natureza escalar. Trata-se de medida do julgamento subjetivo que cada indivíduo faz sobre a qualidade de sua saúde física e mental, com base em critérios pessoais e sociais 2 . No processo de autoavaliação, a comparação com pessoas em piores condições de saúde exerce um papel importante na adaptação dos idosos, pois contribui para minimizar os efeitos negativos da percepção de perdas associadas ao envelhecimento relativas à redução do nível de atividades, de participação social e da própria motivação para o autocuidado em saúde. Trata-se de mecanismo regulador de natureza compensatória, frequentemente mencionado para explicar a divergência, eventualmente observada, entre as avaliações de saúde subjetiva e objetiva 9,10 .

Em estudo de meta-análise, Pinquart 11 observou que bons níveis de saúde física, capacidade funcional e saúde mental relacionavam-se positivamente aos escores de saúde subjetiva. 
Sugere que a percepção mais negativa de idosos mais longevos, em comparação com idosos mais jovens, é reflexo do aumento do número e da gravidade dos problemas de saúde, de limitações da funcionalidade e da diminuição dos recursos materiais e psíquicos para o enfrentamento das doenças que os acometem 11.

A associação entre a saúde subjetiva e o nível socioeconômico é consistentemente relatada na literatura 12,13,14. Ramos 12 observou que os indivíduos de segmentos sociais mais desfavorecidos, principalmente com menor escolaridade, apresentaram pior autoavaliação de saúde. A influência da filiação religiosa na autoavaliação de saúde tem sido analisada por alguns autores, mas os achados não tem sido consistentes 15,16,17.

A literatura aponta que fatores demográficos e socioeconômicos influenciam o estabelecimento de diferentes estilos de vida 18 . Os padrões de comportamento assim desenvolvidos contribuem para a ocorrência ou prevenção de problemas de saúde. A autoavaliação de saúde está associada à adoção de comportamentos de saúde; na literatura, tem sido relatado que a prevalência de comportamentos não saudáveis é maior entre os indivíduos com percepção negativa da saúde 18,19,20.

Uma série de estudos desenvolvidos em outros países tem pesquisado a autoavaliação de saúde em pessoas com 60 anos ou mais $9,13,16,21,22,23$. No entanto, no Brasil, poucos estudos de base populacional analisaram esse indicador especificamente em idosos 24,25,26, havendo vários aspectos da saúde subjetiva desse segmento etário dos brasileiros ainda por investigar. Conhecer os aspectos envolvidos na percepção da saúde pode revelar os subgrupos mais vulneráveis de idosos e subsidiar os serviços de saúde nas iniciativas de promoção de melhor qualidade de vida e saúde.

Considerando a relevância da questão, o presente estudo teve como objetivo analisar a prevalência da saúde autoavaliada como excelente ou muito boa, segundo variáveis demográficas, socioeconômicas e de comportamentos relacionados à saúde, em idosos residentes em Campinas, São Paulo, integrantes de amostra probabilística de um estudo de base populacional.

\section{Material e métodos}

Este estudo é parte de uma pesquisa maior constituída pelo inquérito de saúde de base populacional realizado em 2008-2009, no Município de Campinas (ISACamp 2008-09). O inquérito coletou informações de pessoas não institucionalizadas, residentes na área urbana do município.

\section{Processo amostral do ISACamp 2008-2009}

Para a realização do inquérito, foi fixado como objetivo da pesquisa o estudo de aspectos referentes a três subgrupos da população: adolescentes, adultos e idosos. Dessa forma, foram considerados três grupos de idade (10-19 anos; 20-59 anos e 60 ou mais anos), que constituíram os domínios do estudo. Optou-se pelo sorteio de amostras de tamanhos iguais, de mil pessoas, para cada um dos grupos etários. Com esse tamanho amostral seria possível estimar uma proporção de 0,50 , que corresponde à máxima variabilidade para a frequência dos eventos estudados, com erro de amostragem entre 4 e 5 pontos percentuais, com nível de 95\% de confiança e considerando um efeito de delineamento igual a 2 .

Foi utilizada amostragem por conglomerados, em dois estágios. No primeiro estágio foram sorteados cinquenta setores censitários da área urbana do município com probabilidade proporcional ao tamanho, expresso pelo número de domicílios. A definição do número de setores censitários que deveriam ser sorteados considerou que o total de entrevistas de cada domínio, por setor, não deveria ultrapassar vinte. Nesses cinquenta setores, foi feita pesquisa de campo para arrolamento dos domicílios particulares existentes. No segundo estágio, sorteou-se uma amostra de domicílios nos setores que já haviam sido definidos.

O cálculo do número de domicílios a serem sorteados foi feito com base na média esperada de pessoas por domicílio (razão pessoas/domicílio) em cada grupo de idade, sendo 282.147 o total de domicílios existentes no Município de Campinas, considerando-se os setores censitários urbanos, no ano do Censo Demográfico de 2000 (Instituto Brasileiro de Geografia e Estatística. http://www.ibge.gov.br). As médias de adolescentes, adultos e idosos por domicílio foram: $0,60,1,92$ e 0,32 , respectivamente. Dividiu-se, então, o tamanho desejado da amostra (mil pessoas) por essa razão para estimar o número de domicílios que deveriam ser sorteados para obter tamanho amostral suficiente de cada estrato de idade. Prevendo-se a ocorrência de recusas e de domicílios vagos, foram sorteados $20 \%$ a mais de moradias, elevando os tamanhos de amostra de domicílios para 2.150, 700 e 3.900, respectivos aos três grupos de idade.

Dessa forma, para a obtenção das mil entrevistas de idosos, foram sorteados 3.900 domicílios em que deveriam ser entrevistados todos os idosos que fossem moradores.

Maiores detalhes sobre o processo amostral encontram-se disponíveis na página da Internet: 
http://www.fcm.unicamp.br/centros/ccas/ar quivos/plano_de_amostragem.pdf.

\section{Instrumento de coleta de dados}

As informações foram coletadas por meio de questionário aplicado por entrevistadores treinados e respondido diretamente pelos idosos sorteados. Os conjuntos de variáveis analisadas no presente estudo foram:

1) Autoavaliação da saúde, obtida por meio da pergunta "Em geral, você diria que sua saúde é: excelente, muito boa, boa, ruim ou muito ruim?".

2) Características demográficas: sexo, idade, raça/cor auto referida, situação conjugal, naturalidade, religião e número de moradores no domicílio.

3) Características socioeconômicas: escolaridade, ocupação, renda familiar mensal per capita, número de equipamentos no domicílio e posse de carro e/ou computador.

4) Comportamentos relacionados à saúde, compreendendo:

(a) Atividade física em contexto de lazer, tendo sido classificados como ativos os idosos que a praticavam ao menos 150 minutos por semana, distribuídos, no mínimo, por três dias; como insuficientemente ativos os que a praticavam menos que 150 minutos ou mais de 150 minutos, porém em menos de três dias na semana; como sedentários os que não praticavam qualquer tipo de atividade física de lazer em nenhum dia da semana.

(b) Uso abusivo de bebidas alcoólicas, avaliado pelo Alcohol Use Disorder Identification Test (AUDIT) 27, que é composto por dez questões e identifica o risco de abuso/dependência de álcool quando seu escore (faixa de 0-40) é igual a oito ou mais 28 . Foi também avaliada a frequência do consumo de bebida alcoólica categorizada em: não consome, consome uma a quatro vezes por mês, duas a três vezes por semana e quatro ou mais vezes por semana.

(c) Tabagismo, categorizado em fumante, ex-fumante e não fumante. Foram consideradas como ex-fumantes as pessoas que referiram ter fumado ao menos cem cigarros na vida e haviam cessado com o consumo; foram classificados como fumantes aqueles que persistiam com o consumo do tabaco por ocasião de entrevista.

(d) Índice de massa corporal (IMC), calculado com base em informação autorreferida de peso e altura. Foram usados os pontos de corte adequados para idosos, considerando-se as modificações na composição corporal que ocorrem com o envelhecimento. Os idosos foram categorizados em eutróficos (IMC $22-27 \mathrm{~kg} / \mathrm{m}^{2}$ ) , com baixo pe- so $\left(\right.$ IMC $<22 \mathrm{~kg} / \mathrm{m}^{2}$ ), com sobrepeso (IMC $>27$ a $<30 \mathrm{~kg} / \mathrm{m}^{2}$ ) 29 e obesos (IMC $\geq 30 \mathrm{~kg} / \mathrm{m}^{2}$ ).

(e) Frequência semanal do consumo de frutas e verduras-hortaliças, categorizada em 4 vezes ou mais por semana e menos de 4 vezes por semana.

\section{Análise de dados}

Os dados do inquérito foram digitados em banco de dados desenvolvido com o uso do software Epidata, versão 3.1 (Epidata Assoc., Odense, Dinamarca) e submetidos à avaliação de consistência. Para as análises deste estudo, foram produzidas estimativas de prevalências e dos intervalos de 95\% de confiança (IC95\%). As associações entre as variáveis independentes e a autoavaliação de saúde foram analisadas pelo teste Qui-quadrado. Também foram usadas as análises de regressão simples e múltipla de Poisson para estimar razões de prevalência (RP) brutas e ajustadas. Foi desenvolvido modelo de regressão múltipla de Poisson em duas etapas. Na primeira, foram introduzidas as variáveis demográficas e socioeconômicas que apresentaram um nível de significância menor de $20 \%$ ( $\mathrm{p}<0,20)$ na associação com a variável dependente; permaneceram no modelo aquelas com $p<0,05$. Na segunda, além das variáveis que permaneceram na etapa anterior, foram acrescidas as de comportamentos relacionados à saúde, com $\mathrm{p}<0,20$ na análise univariada; mantiveramse na segunda etapa do modelo aquelas com $\mathrm{p}<0,05$. A análise dos dados foi realizada com os comandos svy do software Stata versão 11.0 (Stata Corp., Chicago, Estados Unidos), utilizando-se as ponderações decorrentes do desenho amostral e considerando-se a existência das unidades primárias de amostragem.

\section{Procedimentos éticos}

O presente estudo foi aprovado pelo Comitê de Ética em Pesquisa da Faculdade de Ciências Médicas da Universidade Estadual de Campinas, em adendo ao parecer no. 079/2007, que havia aprovado o projeto do ISACamp 2008-2009. Todos os participantes assinaram Termo de Consentimento Livre e Esclarecido.

\section{Resultados}

Houve perda de 6,5\% dos domicílios que haviam sido sorteados para as entrevistas com idosos em virtude da impossibilidade de encontrar um morador ou da recusa deste em arrolar os indivíduos que moravam no domicílio. Entre os 1.558 
idosos identificados nos domicílios sorteados, $2,3 \%$ recusaram-se a participar da pesquisa e, em 5,8\% dos casos, a informação foi dada por um cuidador ou familiar responsável. Dessa forma, neste estudo foram analisados os dados de 1.432 idosos, dos quais $57,2 \%$ eram mulheres. A idade média da população estudada foi 69,9 anos (IC95\%: 69,2-70,6). Na Tabela 1, observase que $76,1 \%$ referiram ser de cor branca, $57,6 \%$ eram casados, $46,2 \%$ nasceram em outros municípios do Estado de São Paulo, 39\% moravam com apenas uma pessoa no domicílio e $16,7 \%$ moravam sozinhos. Em relação à escolaridade, $63,4 \%$ tinham quatro anos ou menos de estudo,
$39,2 \%$ tinham renda familiar per capita inferior a um salário mínimo e 78,8\% não tinham trabalho remunerado (Tabela 2 ).

A prevalência estimada da autoavaliação de saúde excelente ou muito boa foi de $24,6 \%$; 8,2\% (IC95\%: 6,2-10,0) dos participantes avaliaram sua saúde como excelente; 16,4\% (IC95\%: 13,5-19,9), como muito boa; $64,5 \%$ (IC95\%: 59,469,1), como boa; $10 \%$ (IC95\%: 8,1-12,0), como ruim; e 0,9\% (IC95\%: 0,5-1,7), como muito ruim. Nas RP ajustadas por sexo e idade, observou-se que os indivíduos com 70 a 79 anos $(\mathrm{RP}=0,73)$, os não naturais do Estado de São Paulo (RP = 0,63 ), aqueles que moravam com duas ou mais

\section{Tabela 1}

Prevalência e razão de prevalência (RP) de saúde autoavaliada como excelente ou muito boa segundo variáveis sociodemográficas. Inquérito de Saúde de Campinas (ISACamp), 2008-2009.

\begin{tabular}{|c|c|c|c|c|c|}
\hline Variáveis & $\mathrm{n}$ & $\%$ & Prevalência & $\begin{array}{c}\mathrm{RP} \\
\text { (IC95\%) }\end{array}$ & $\begin{array}{l}\text { RP ajustada * } \\
\text { (IC95\%) }\end{array}$ \\
\hline Sexo & & & $p=0,3364$ & & \\
\hline Feminino & 852 & 57,2 & 23,7 & 1,00 & 1,00 \\
\hline Masculino & 580 & 42,8 & 25,9 & $1,09(0,90-1,32)$ & $1,08(0,89-1,30)$ \\
\hline Total & 1.432 & 100,0 & 24,6 & & \\
\hline Faixa etária (anos) & & & $p=0,0203$ & & \\
\hline $60-69$ & 800 & 56,0 & 27,6 & 1,00 & 1,00 \\
\hline $70-79$ & 470 & 32,7 & 20,3 & $0,73(0,58-0,92)$ & $0,73(0,58-0,93)$ \\
\hline 80 e mais & 162 & 11,3 & 22,6 & $0,81(0,59-1,12)$ & $0,82(0,60-1,13)$ \\
\hline Situação conjugal & & & $p=0,2473$ & & \\
\hline Viúvo & 452 & 28,2 & 21,0 & 1 & 1,00 \\
\hline Casado & 848 & 57,6 & 24,7 & $1,15(0,90-1,48)$ & $1,04(0,81-1,33)$ \\
\hline Separado & 120 & 7,9 & 30,3 & $1,41(1,03-1,92)$ & $1,28(0,94-1,74)$ \\
\hline Solteiro & 100 & 6,3 & 26,4 & $1,30(0,85-2,00)$ & $1,25(0,83-1,91)$ \\
\hline Cor/Raça & & & $p=0,1224$ & & \\
\hline Branca & 1.084 & 76,1 & 26,3 & 1,00 & 1,00 \\
\hline Não branca & 346 & 23,9 & 19,5 & $0,74(0,50-1,11)$ & $0,71(0,48-1,06)$ \\
\hline Naturalidade & & & $p=0,0438$ & & \\
\hline Campinas & 295 & 20,7 & 30,6 & 1,00 & 1,00 \\
\hline Outro município do Estado de São Paulo & 660 & 46,2 & 25,7 & $0,84(0,60-1,16)$ & $0,84(0,61-1,16)$ \\
\hline Outro estado & 476 & 33,1 & 19,5 & $0,64(0,43-0,94)$ & $0,63(0,43-0,93)$ \\
\hline Religião & & & $p=0,0007$ & & \\
\hline Católica & 950 & 66,5 & 22,7 & 1,00 & 1,00 \\
\hline Evangélica & 316 & 21,7 & 20,8 & $0,91(0,64-1,29)$ & $0,90(0,64-1,28)$ \\
\hline Sem religião & 83 & 6,1 & 45,0 & $1,97(1,38-2,81)$ & $1,90(1,36-2,65)$ \\
\hline Outras & 80 & 5,7 & 38,4 & $1,68(1,17-2,42)$ & $1,66(1,16-2,38)$ \\
\hline Número de moradores no domicílio & & & $p=0,0343$ & & \\
\hline 1 & 242 & 16,7 & 31,6 & 1,00 & 1,00 \\
\hline 2 & 552 & 39,0 & 24,7 & $0,78(0,59-1,02)$ & $0,72(0,56-0,93)$ \\
\hline$\geq 3$ & 638 & 44,3 & 22,0 & $0,69(0,53-0,91)$ & $0,63(0,48-0,82)$ \\
\hline
\end{tabular}

IC95\%: intervalo de 95\% de confiança.

* Ajustada por idade e sexo. 
Prevalência e razão de prevalência (RP) de saúde autoavaliada como excelente ou muito boa segundo variáveis socioeconômicas. Inquérito de Saúde de Campinas (ISACamp), 2008-2009.

\begin{tabular}{|c|c|c|c|c|c|}
\hline Variáveis & $\mathrm{n}$ & $\%$ & Prevalência & $\begin{array}{c}\mathrm{RP} \\
\text { (IC95\%) }\end{array}$ & $\begin{array}{l}\text { RP ajustada * } \\
\text { (IC95\%) }\end{array}$ \\
\hline Escolaridade do indivíduo (anos) & & & $p<0,0001$ & & \\
\hline Nunca & 242 & 16,4 & 8,3 & 1,00 & 1,00 \\
\hline $1-4$ & 682 & 47,0 & 19,9 & $2,39(1,57-3,64)$ & $2,38(1,55-3,63)$ \\
\hline $5-8$ & 166 & 11,5 & 33,4 & $4,02(2,59-6,23)$ & $3,97(2,57-6,15)$ \\
\hline$\geq 9$ & 339 & 25,1 & 40,5 & $4,87(3,06-7,74)$ & $4,86(3,03-7,80)$ \\
\hline Renda familiar per capita (salários mínimos) & & & $p<0,0001$ & & \\
\hline$<1$ & 572 & 39,2 & 16,4 & 1,00 & 1,00 \\
\hline $1-3$ & 591 & 41,0 & 25,3 & $1,54(1,21-1,97)$ & $1,51(1,18-1,93)$ \\
\hline$>3$ & 269 & 19,8 & 39,8 & $2,43(1,83-3,22)$ & $2,37(1,79-3,13)$ \\
\hline Trabalho remunerado & & & $p=0,0036$ & & \\
\hline Não & 1.136 & 78,8 & 22,4 & 1,00 & 1,00 \\
\hline Sim & 295 & 21,2 & 32,6 & $1,45(1,14-1,85)$ & $1,35(1,03-1,79)$ \\
\hline Número de equipamentos no domicílio & & & $\mathrm{p}<0,0001$ & & \\
\hline $1-5$ & 291 & 19,8 & 14,7 & 1,00 & 1,00 \\
\hline $6-10$ & 583 & 40,2 & 20,3 & $1,38(0,96-1,98)$ & $1,35(0,95-1,92)$ \\
\hline 11 e mais & 558 & 40,0 & 34,0 & $2,31(1,55-3,44)$ & $2,22(1,50-3,29)$ \\
\hline Possui computador & & & $p<0,0001$ & & \\
\hline Não & 942 & 64,9 & 18,9 & 1,00 & 1,00 \\
\hline Sim & 490 & 35,1 & 35,3 & $1,86(1,45-2,39)$ & $1,81(1,41-2,32)$ \\
\hline Possui carro & & & $p<0,0001$ & & \\
\hline Não & 690 & 47,4 & 19,9 & 1,00 & 1,00 \\
\hline Sim & 741 & 52,6 & 29,0 & $1,45(1,22-1,73)$ & $1,41(1,18-1,70)$ \\
\hline
\end{tabular}

IC95\%: intervalo de 95\% de confiança.

* Ajustada por idade e sexo.

pessoas e os católicos (em relação aos sem religião ou com outra religião) apresentaram menores prevalências de saúde autoavaliada como excelente ou muito boa (Tabela 1).

Idosos com um ano ou mais de escolaridade, com renda de um salário mínimo ou mais, que dispunham de 11 ou mais equipamentos no domicílio, que possuíam carro ou computador e os que tinham trabalho remunerado apresentaram maior prevalência de saúde autoavaliada como excelente ou muito boa (Tabela 2). A prevalência apresentou gradiente crescente com o aumento da escolaridade e com o aumento da renda, atingindo RP de 4,86 no segmento de nove ou mais anos de escolaridade e RP de 2,37 no estrato de renda superior a três salários mínimos.

Os indivíduos que consumiam bebida alcoólica de uma a quatro vezes por mês, os que não eram sedentários no lazer e os que consumiam frutas e verduras quatro vezes ou mais por semana apresentaram maior prevalência de autoava- liação de saúde excelente/muito boa, sendo esta prevalência significativamente menor entre os obesos (Tabela 3).

Os resultados da análise de regressão múltipla de Poisson identificaram maior prevalência de saúde excelente/muito boa entre os indivíduos sem religião, com um ano de escolaridade ou mais, renda per capita igual a ou acima de um salário mínimo, que moravam sozinhos, que tinham computador em casa, que consumiam bebida alcoólica uma a quatro vezes por mês, que eram ativos ou insuficientemente ativos, que consumiam frutas e verduras quatro vezes ou mais por semana e que não eram obesos (Tabela 4).

\section{Discussão}

Observou-se predominância de mulheres na população estudada, assim como vem sendo 
Prevalência e razão de prevalência (RP) de saúde autoavaliada como excelente ou muito boa segundo comportamentos relacionados à saúde. Inquérito de Saúde de Campinas (ISACamp), 2008-2009.

\begin{tabular}{|c|c|c|c|c|c|}
\hline Variáveis & $\mathrm{n}$ & $\%$ & Prevalência & $\begin{array}{l}\text { RP bruta } \\
\text { (IC95\%) }\end{array}$ & $\begin{array}{l}\text { RP ajustada * } \\
\text { (IC95\%) }\end{array}$ \\
\hline Tabagismo & & & $p=0,7607$ & & \\
\hline Nunca fumou & 973 & 67,3 & 25,2 & 1,00 & 1,00 \\
\hline Ex-fumante & 166 & 11,7 & 23,0 & $0,91(0,68-1,21)$ & $0,85(0,62-1,16)$ \\
\hline Fumante & 292 & 21,0 & 23,5 & $0,93(0,68-1,28)$ & $0,90(0,63-1,29)$ \\
\hline AUDIT & & & $p=0,4588$ & & \\
\hline Negativo & 1.372 & 95,7 & 24,9 & 1,00 & 1,00 \\
\hline Positivo & 59 & 4,3 & 20,3 & $0,81(0,47-1,42)$ & $0,73(0,42-1,28)$ \\
\hline Frequência de consumo de álcool & & & $p=0,0001$ & & \\
\hline Não bebe & 991 & 68,2 & 21,0 & 1,00 & 1,00 \\
\hline $1-4$ vezes/mês & 299 & 21,3 & 36,4 & $1,73(1,38-2,17)$ & $1,70(1,35-2,14)$ \\
\hline 2-3 vezes/semana & 73 & 5,4 & 24,0 & $1,14(0,75-1,73)$ & $1,14(0,75-1,73)$ \\
\hline 4 ou mais vezes/semana & 68 & 5,0 & 25,6 & $1,21(0,76-1,95)$ & $1,19(0,73-1,96)$ \\
\hline Atividade física de lazer & & & $p<0,0001$ & & \\
\hline Sedentário & 965 & 66,9 & 19,0 & 1,00 & 1,00 \\
\hline Insuficientemente ativo & 156 & 10,9 & 33,6 & $1,77(1,29-2,42)$ & $1,73(1,26-2,38)$ \\
\hline Ativo & 311 & 22,2 & 37,3 & $1,96(1,50-2,56)$ & $1,92(1,47-2,52)$ \\
\hline Frutas/Verduras-hortaliças (frequência) & & & $p<0,0001$ & & \\
\hline$\geq 4$ vezes/semana & 898 & 62,7 & 28,9 & 1,00 & 1,00 \\
\hline$<4$ dias/semana & 534 & 37,3 & 17,5 & $0,60(0,49-0,75)$ & $0,59(0,48-0,73)$ \\
\hline $\mathrm{IMC}\left(\mathrm{kg} / \mathrm{m}^{2}\right)$ & & & $p=0,0053$ & & \\
\hline $22-27$ & 647 & 47,2 & 28,8 & 1,00 & 1,00 \\
\hline$<22$ & 263 & 18,8 & 23,5 & $0,81(0,62-1,07)$ & $0,83(0,64-1,07)$ \\
\hline$>27 a<30$ & 265 & 19,1 & 25,1 & $0,87(0,67-1,13)$ & $0,86(0,66-1,11)$ \\
\hline$\geq 30$ & 208 & 14,8 & 14,5 & $0,50(0,33-0,76)$ & $0,50(0,32-0,75)$ \\
\hline
\end{tabular}

AUDIT: Alcohol Use Disorder Identification Test; IC95\%: intervalo de 95\% de confiança; IMC: índice de massa corporal.

* Ajustada por idade e sexo.

notado em outros estudos com a população idosa 24,25. O número de mulheres idosas no Brasil é maior do que o de homens; em 2003, essa proporção era de 55,9\% e 44,1\%, respectivamente 30 .

A proporção de idosos analfabetos foi de $16,4 \%$, percentual que é menor que o do país, refletindo melhor nível de desenvolvimento socioeconômico e menor número de indivíduos vivendo em condições de alta vulnerabilidade social. No Brasil, em 2007, o percentual de idosos analfabetos foi $32,2 \%$, enquanto na Região Sudeste foi $22,8 \% 31$.

A maior parte dos idosos deste estudo vive com renda familiar per capita de um a três salários mínimos (41\%), e 39,2\% vivem com renda inferior a um salário mínimo. O rendimento familiar per capita é um indicador de acesso a bens essenciais, especialmente em situação de ausência ou insuficiência de políticas sociais que garantam moradia, transporte e atendimento à saúde adequado e de qualidade 30 .

Neste estudo, a prevalência da autoavaliação de saúde excelente ou muito boa foi de $24,6 \%$. Embora tendo utilizado diferentes categorias de respostas e havendo diferenças socioeconômicas entre as cidades, a pesquisa realizada no Município de Bambuí, Minas Gerais, com 1.516 idosos, encontrou resultado similar, com prevalência de $24,7 \%$ de saúde muito boa ou boa 24 .

Não foi observada diferença estatisticamente significativa entre as frequências de homens e de mulheres que avaliaram a saúde de forma positiva ou negativa, dado que contraria a ocorrência mais comum de pior autoavaliação de saúde entre as mulheres 15,19,32,33,34. Com base em dados de um estudo longitudinal com 59 anos de seguimento, McCullough \& Laurenceau 15 observaram que a diferença entre os gêneros na autoavaliação 
Dois modelos de regressão múltipla de Poisson. Inquérito de Saúde de Campinas (ISACamp), 2008-2009.

\begin{tabular}{|c|c|c|}
\hline \multirow[t]{2}{*}{ Variáveis } & Primeira etapa & Segunda etapa \\
\hline & RP ajustada * (IC95\%) & RP ajustada ** (IC95\%) \\
\hline \multicolumn{3}{|l|}{ Religião } \\
\hline Católica & 1,00 & 1,00 \\
\hline Evangélica & $1,13(0,81-1,58)$ & $1,20(0,88-1,63)$ \\
\hline Sem religião & $1,70(1,35-2,13)$ & $1,63(1,31-2,04)$ \\
\hline Outras & $1,33(0,94-1,88)$ & $1,30(0,94-1,81)$ \\
\hline \multicolumn{3}{|c|}{ Escolaridade do indivíduo (anos) } \\
\hline Nunca & 1,00 & 1,00 \\
\hline $1-4$ & $2,08(1,37-3,17)$ & $1,97(1,25-3,10)$ \\
\hline $5-8$ & $2,98(1,95-4,55)$ & $2,74(1,71-4,40)$ \\
\hline$\geq 9$ & $3,21(2,01-5,12)$ & $2,81(1,71-4,63)$ \\
\hline \multicolumn{3}{|c|}{ Renda familiar per capita (salários mínimos) } \\
\hline$<1$ & 1,00 & 1,00 \\
\hline $1-3$ & $1,34(1,07-1,67)$ & $1,23(0,97-1,54)$ \\
\hline$>3$ & $1,46(1,14-1,86)$ & $1,27(0,97-1,66)$ \\
\hline \multicolumn{3}{|c|}{ Número de moradores no domicílio } \\
\hline 1 & 1,00 & 1,00 \\
\hline 2 & $0,73(0,57-0,94)$ & $0,73(0,58-0,92)$ \\
\hline$\geq 3$ & $0,71(0,53-0,95)$ & $0,74(0,56-0,98)$ \\
\hline \multicolumn{3}{|l|}{ Possui computador } \\
\hline Não & 1,00 & 1,00 \\
\hline Sim & $1,41(1,10-1,80)$ & $1,35(1,07-1,71)$ \\
\hline \multicolumn{3}{|l|}{ Frequência de álcool } \\
\hline Não bebe & & 1,00 \\
\hline $1-4$ vezes/mês & & $1,31(1,06-1,62)$ \\
\hline $2-3$ vezes/semana & & $0,89(0,62-1,28)$ \\
\hline 4 ou mais vezes/semana & & $1,13(0,72-1,77)$ \\
\hline \multicolumn{3}{|l|}{ Atividade física de lazer } \\
\hline Sedentário & & 1,00 \\
\hline Insuficientemente ativo & & $1,40(1,02-1,93)$ \\
\hline Ativo & & $1,42(1,10-1,82)$ \\
\hline \multicolumn{3}{|c|}{ Frutas/Verduras-hortaliças (frequência) } \\
\hline$\geq 4$ vezes/semana & & 1,00 \\
\hline$<4$ dias/semana & & $0,72(0,59-0,88)$ \\
\hline \multicolumn{3}{|l|}{$\mathrm{IMC}\left(\mathrm{kg} / \mathrm{m}^{2}\right)$} \\
\hline $22-27$ & & 1,00 \\
\hline$<22$ & & $0,94(0,76-1,16)$ \\
\hline$<27 a<30$ & & $0,93(0,75-1,15)$ \\
\hline$\geq 30$ & & $0,60(0,42-0,87)$ \\
\hline
\end{tabular}

IC95\%: intervalo de 95\% de confiança; RP: razão de prevalência.

* Ajustada pelas variáveis demográficas e socioeconômicas;

** Ajustada por todas as variáveis da tabela.

de saúde diminuiu com o avançar da idade e desapareceu entre os idosos de 80 anos e mais.

A piora da autoavaliação de saúde com o avançar da idade é consistentemente observada $1,23,33,35$, sendo interpretada como fruto do au- mento das comorbidades e das incapacidades funcionais 33,36. Neste estudo, a diminuição da prevalência da autoavaliação da saúde como excelente ou muito boa com a idade não ocorreu no segmento de 80 anos e mais. Os idosos mais 
jovens, entre 60-69 anos, apresentaram prevalência significativamente maior de saúde excelente/ muito boa quando comparados aos idosos com 70-79 anos na análise univariada, mas a significância não persistiu na análise ajustada por variáveis socioeconômicas.

A forte associação da autoavaliação da saúde com o nível de escolaridade é uma constatação frequente na literatura 1,13,23,24,34. O mesmo foi verificado neste estudo, que detectou aumento progressivo e intenso da RP dos indivíduos que avaliaram a própria saúde como excelente ou muito boa conforme maior escolaridade. Uma pesquisa com idosos, realizada com base nos dados do projeto Saúde, Bem-estar e Envelhecimento na América Latina e Caribe (SABE), observou que os indivíduos com um a quatro anos de escolaridade tiveram 0,72 razão de chance, e aqueles com cinco anos ou mais apresentaram 0,38 razão de chance de relatar pior saúde percebida quando comparados àqueles sem escolaridade 26. Essa relação provavelmente decorre da associação entre nível de escolaridade e o acesso a oportunidades sociais ao longo de toda a vida, entre elas o acesso à informação, aos serviços de saúde e a melhores condições de vida.

Em estudo transversal de base populacional realizado nos Estados Unidos, foi observado que indivíduos pertencentes aos estratos de nível socioeconômico mais baixos relataram pior autoavaliação da saúde do que os pertencentes aos níveis mais altos; por sua vez, indivíduos de 35-44 anos de nível socioeconômico inferior avaliaram mais negativamente a própria saúde do que os de 65 anos e mais que pertenciam ao melhor nível socioeconômico 14. No estudo norte-americano, a renda foi preditor mais robusto de saúde autorrelatada do que a escolaridade, diferentemente do que ocorreu na presente investigação, em que renda e escolaridade persistiram no modelo final, associadas, inclusive, com a escolaridade, apresentando $\mathrm{RP}$ mais elevada $(\mathrm{RP}=4,86)$ do segmento de maior escolaridade em relação aos analfabetos do que a observada entre os estratos de renda $(R P=2,37)$ do segmento de maior renda em relação ao de menor renda.

A ausência de associação entre autoavaliação de saúde e cor ou raça autorreferidas é compatível com os dados de outro estudo brasileiro 1 , porém diverge do observado em estudos realizados em outros países 21,37, que detectaram associação entre a autoavaliação de saúde e raça. É possível que, no Brasil, baixos níveis de renda e de escolaridade sejam determinantes mais fortes do estado de saúde e das concepções sobre bem-estar do que valores culturais associados à raça. A parcela da população de baixa renda e baixa escolaridade, independen- temente da raça, seria igualmente afetada pelas precárias condições de vida e de atendimento a necessidades de saúde, o que se reflete nas autoavaliações de saúde.

Ter computador em casa, que é também um indicador de bem-estar econômico, significou ter $\mathrm{RP}=1,35$ de relatar saúde excelente ou muito boa em comparação com o grupo que não possuía este equipamento, mesmo após ajuste por escolaridade e renda. De acordo com uma pesquisa sobre os significados que idosos atribuem à saúde, estes a definem principalmente como ter autonomia, ser capaz de agir sobre o ambiente ${ }^{38}$. Sob esta ótica, pode-se supor que os idosos que utilizam o computador e, em especial, têm acesso à Internet, tenderiam a avaliar mais positivamente a própria saúde porque teriam mais condições para expressar capacidades e competências e para integrar-se no contexto social. Outras investigações precisam ser feitas para avaliar a consistência dessa associação, pois ela sinaliza alternativas de promoção de bem-estar e saúde dos idosos com a ampliação do acesso a esse tipo de equipamento.

Maior prevalência de saúde muito boa/excelente foi encontrada nos indivíduos que relataram não ter religião. Estudo transversal, com 499 idosos avaliando a associação de religião com a qualidade de vida no último ano de vida, observou resultado semelhante ao do presente trabalho 17 , assim como pesquisa realizada com idosos da América Latina e do Caribe, segundo a qual filiação religiosa estava associada com pior avaliação de saúde, embora essa associação não se mantivesse após ajuste por capacidade funcional 16. O achado é interessante por contrariar a crença comumente difundida de associação positiva entre religião e saúde. Os resultados da literatura quanto a essa questão não são consistentes. Estudo que avaliou a filiação religiosa e a trajetória da autoavaliação de saúde na vida adulta observou que as pessoas que declararam ter alguma religião apresentaram melhores escores de saúde subjetiva ${ }^{15}$. Este é um tema que necessita ser mais investigado e autores têm ressaltado a importância de considerar nas pesquisas diferentes medidas do envolvimento religioso e diferentes dimensões da religiosidade.

Os idosos que moravam sozinhos apresentaram melhor autoavaliação de saúde quando comparados àqueles que vivem com duas ou mais pessoas, semelhante ao observado em outros estudos transversais de base populacional realizados com idosos brasileiros 24,25,26. Esta associação tem sido atribuída ao fato de que aqueles que vivem sós estariam em melhores condições físicas ${ }^{25}$. Além disso, é provável que idosos vivendo sem companhia tenham melho- 
res condições econômicas e não necessitem dar apoio material ou de cuidado a outros membros da família; dessa forma, podem tratar melhor da própria saúde 39 .

Os idosos que faziam uso de bebida alcoólica de uma a quatro vezes por mês apresentaram melhor autoavaliação de saúde comparados aos abstêmios. Outros estudos transversais observaram maior prevalência de ativos no lazer $40 \mathrm{e}$ melhor qualidade de vida 41 nos idosos que consumiam bebidas alcoólicas moderadamente. Os idosos que bebem em pouca quantidade têm condição melhor de saúde, o que os possibilita continuar ingerindo bebida alcoólica; há ainda o efeito benéfico da ingestão moderada de álcool, principalmente como protetor nas coronariopatias 42 . No entanto, o abuso de álcool pode ter consequências prejudiciais à saúde 42 .

A adoção de um estilo de vida não sedentário calcado na prática regular de atividades físicas aumenta a possibilidade de reduzir diretamente o risco para o desenvolvimento da maior parte das doenças crônico-degenerativas 43 . A prática de exercício no período de lazer constitui um indicador de incorporação de estilos de vida mais saudáveis 40 . Com a adoção de um estilo de vida ativo, o idoso pode controlar e evitar alguns sintomas de doenças, melhorar ou manter a aptidão física e melhorar a capacidade de realizar suas atividades. Além dos benefícios fisiológicos, a atividade física propicia benefícios psicológicos e sociais, o que influencia a autoavaliação da saúde 44. É importante ressaltar que o nosso estudo encontrou que ser insuficientemente ativo já tem associação com melhor saúde subjetiva, o que apontaria que entre os idosos o estímulo a algum nível de atividade física, mesmo que inferior às recomendações, já poderia ter resultados satisfatórios quanto ao bem-estar. Por outro lado, idosos em melhores condições de saúde podem estar mais motivados e capacitados para as práticas da atividade física. De fato, delineamentos transversais estão sujeitos ao viés de causalidade reversa, não permitindo identificar associações causais.

O consumo de frutas e verduras apresentou associação com autoavaliação da saúde, repli- cando dados de outros estudos brasileiros 19,24 com a população idosa 19 e adulta 24 . A qualidade da dieta é um fator importante nos hábitos saudáveis do indivíduo e consequentemente na melhoria da qualidade de vida.

Outros estudos transversais mostraram associação da autoavaliação de saúde com o IMC, encontrando pior autoavaliação entre os obesos 33,45. O excesso de tecido adiposo no organismo pode estar relacionado direta ou indiretamente com doenças cardiovasculares, osteomusculares e neoplásicas; além disso, podem causar incapacidade funcional e problemas psicológicos e de interação social 46 .

Alguns dos achados do presente estudo, como a associação negativa com a filiação religiosa e com a obesidade, e a associação positiva com a posse de computador, correspondem a variáveis que não haviam sido analisadas anteriormente em outros trabalhos brasileiros de base populacional realizados sobre a autoavaliação de saúde de idosos. São resultados interessantes que sinalizam a necessidade de outras investigações sobre esses temas.

Mesmo reconhecendo a possível discordância entre avaliações subjetivas e objetivas e o fato de aquelas estarem sujeitas a vieses pessoais e sociais, a saúde percebida é um bom preditor de morbidade, incapacidade e mortalidade. Tratase, portanto, de um indicador útil aos planejadores e executores de políticas de saúde.

Os resultados deste estudo, em que a autoavaliação de saúde como excelente ou muito boa destaca-se entre os idosos com melhor escolaridade e renda, que moravam sozinhos e que tinham computador em casa, indicam a presença da desigualdade social no estado de saúde; logo, é necessário maior empenho nos programas de saúde e bem-estar social voltados para os segmentos socioeconômicos menos favorecidos. Os achados também apontam a existência de forte associação da autoavaliação de saúde com comportamentos relativos à saúde, como consumo frequente de frutas e verduras e uso moderado do álcool, o que reforça a necessidade de estratégias de promoção de hábitos saudáveis entre os idosos. 


\section{Resumo}

Nesta pesquisa, analisou-se a autoavaliação da saúde em idosos segundo variáveis demográficas, socioeconômicas e de comportamentos relacionados à saúde. Trata-se de estudo transversal de base populacional, com amostra por conglomerados, que utilizou dados de inquérito realizado em Campinas, São Paulo, Brasil (ISACamp 2008/2009). Foram estimadas razões de prevalências ajustadas por meio de regressão múltipla de Poisson. Participaram do estudo 1.432 idosos. A prevalência de saúde excelente/muito boa foi $24,6 \%$ e significativamente mais elevada nos idosos com maior escolaridade, maior renda, sem religião, que moravam sozinhos, tinham computador em casa, consumiam bebida alcoólica de uma a quatro vezes por mês, praticavam atividade física no lazer, não eram obesos $e$ consumiam frutas e verduras quatro vezes ou mais por semana. Alguns dos achados são pouco encontrados na literatura e sinalizam temas relevantes para novas investigações. Os resultados apontam para a necessidade de maior atenção aos segmentos socialmente mais vulneráveis e do desenvolvimento de estratégias de promoção de hábitos saudáveis entre os idosos.

Saúde do Idoso; Inquéritos Epidemiológicos; Autoavaliação

\section{Referências}

1. Dachs NW, Santos APR. Auto-avaliação do estado de saúde no Brasil: análise dos dados da PNAD/2003. Ciênc Saúde Coletiva 2006; 11:887-94.

2. Neri AL. Qualidade de vida na velhice e subjetividade. In: Neri AL, organizadora. Qualidade de vida na velhice: enfoque multidisciplinar. Campinas: Editora Alínea; 2007. p. 13-59.

3. Blazer DG. How do you feel about...? Health outcomes in late life and self-perceptions of health and well-being. Gerontologist 2008; 48:415-22.

4. Martin LG, Schoeni RF, Freedman VA, Andreski P. Feeling better? Trends in general health status. J Gerontol B Psychol Sci Soc Sci 2007; 62:S11-21.

5. Bernard SL, Kincade JE, Konrad TR, Arcury TA, Rabiner DJ, Woomert A, et al. Predicting mortality from community surveys of older adults: the importance of self-rated functional ability. J Gerontol B Psychol Sci Soc Sci 1997; 52:S155-63.

\section{Colaboradores}

F. S. A. Borim realizou a proposta do artigo, revisão da literatura, análise e interpretação dos dados, redação do texto. M. B. A. Barros e A. L. Neri colaboraram com a análise e interpretação dos dados, redação e revisão crítica do conteúdo intelectual.

\section{Agradecimentos}

Ao Conselho Nacional de Desenvolvimento Científico e Tecnológico (CNPq; processo $\mathrm{n}^{\mathrm{o}}$. 409747/2006-8), pelo financiamento da pesquisa e bolsa produtividade de $\mathrm{M}$. B. A. Barros. À Secretaria Municipal de Saúde de Campinas e à Secretaria de Vigilância em Saúde, Ministério da Saúde, pelo apoio para o trabalho de campo. À Fundação de Amparo à Pesquisa do Estado de São Paulo (FAPESP), pela bolsa de doutorado de F. S. A. Borim.
6. Shirom A, Toker S, Berliner S, Shapira I, Melamed S. The effects of physical fitness and feeling vigorous on self-rated health. Health Psychol 2008; 27:567-75.

7. Ilder EL, Benyamini Y. Self-rated health and mortality: a review of twenty-seven community studies. J Health Soc Behav 1997; 38:21-37.

8. Sargent-Cox KA, Anstey KJ, Luszcz MA. The choice of self-rated health measures matter when predicting mortality: evidence from 10 years follow-up of the Australian longitudinal study of ageing. BMC Geriatr 2010; 10:18.

9. Liang J, Shaw BA, Krause N, Bennett JM, Kobayashi E, Fukaya T, et al. How does self-assessed health change with age? A study of older adults in Japan. J Gerontol B Psychol Sci Soc Sci 2005; 60:S224-32. 
10. Cheng ST, Fung H, Chan A. Maintaining self-rated health through social comparison in old age. J Gerontol B Psychol Sci Soc Sci 2007; 62:S277-85.

11. Pinquart $M$. Correlates of subjective health in older adults: a meta-analysis. Psychol Aging 2001; 16:414-26.

12. Ramos M. Impact of socioeconomic status on Brazilian elderly health. Rev Saúde Pública 2007; 41:616-24.

13. Feinglass J, Lin S, Thompson J, Sudano J, Dunlop $\mathrm{D}$, Song J, et al. Baseline health, socioeconomic status and 10-year mortality among older middleaged Americans: findings from the health and retirement study, 1992-2002. J Gerontol B Psychol Sci Soc Sci 2007; 62:S209-17.

14. Robert AS, Cherepanov D, Palta M, Dunham NC, Feeny D, Fryback DG. Socioeconomic status and age variations in health-related quality of life: results from the National Health Measurement Study. J Gerontol B Psychol Sci Soc Sci 2009; 64:S378-89.

15. McCullough ME, Laurenceau JP. Religiousness and the trajectory of self-rated health across adulthood. Pers Soc Psychol Bull 2005; 31:560-73.

16. Reyes-Ortiz CA, Pelaez M, Koenig HG, Mulligan T. Religiosity and self-rated health among Latin American and Caribbean elders. Int J Psychiatry Med 2007; 37:425-43.

17. Idler EL, McLaughlin J, Kasl S. Religion and the quality of life in last year of life. J Gerontol B Psychol Sci Soc Sci 2009; 64:S528-37.

18. Manderbacka K, Lundberg O, Martikainen P. Do risk factors and health behaviours contribute to selfratings of health? Soc Sci Med 1999; 48:1713-20.

19. Barros MBA, Zanchetta LM, Moura EC, Malta DC. Auto-avaliação da saúde e fatores associados, Brasil, 2006. Rev Saúde Pública 2009; 43 Suppl 2:27-37.

20. Barreto SM, Figueiredo RC. Doença crônica, auto-avaliação de saúde e comportamento de risco: diferença de gênero. Rev Saúde Pública 2009; 43 Suppl 2:38-47.

21. McMullen CK, Luborsky MR. Self-rated health appraisal as cultural and identity process: African American elders' health and evaluative rationales. Gerontologist 2006; 46:431-8.

22. Menec VH, Shooshtari S, Lambert P. Ethnic differences in self-rated health among older adults. J Aging Health 2007; 19:62-86.

23. Miller TR, Wolinsky FD. Self-rated health trajectories and mortality among older adults. J Gerontol B Psychol Sci Soc Sci 2007; 62:S22-7.

24. Lima-Costa MF, Firmo JOA, Uchôa E. A estrutura da auto-avaliação da saúde entre idosos: projeto Bambuí. Rev Saúde Pública 2004; 38:827-34.

25. Lebrão ML, Laurenti R. Saúde, bem-estar e envelhecimento: o estudo SABE no Município de São Paulo. Rev Bras Epidemiol 2005; 8:127-41.

26. Alves LC, Rodrigues RN. Determinantes da autopercepção de saúde entre idosos do Município de São Paulo, Brasil. Rev Panam Salud Pública 2005; 17:333-41.

27. Dawson DA, Grant BF, Stinson FS, Zhou Y. Effectiveness of the derived alcohol us disorders identification test (AUDIT-C) in screening for alcohol use disorders and risk drinking in the USD general population. Alcohol Clin Exp Res 2005; 29:844-54.
28. Lima CT, Freire ACC, Silva APB, Teixeira RM, Farrel $\mathrm{M}$, Prince M. Concurrent and construct validity of the AUDIT in an urban Brazilian sample. Alcohol Alcohol 2005; 40:584-9.

29. Cervi A, Franceschini SC, Priore SE. Análise crítica do uso do índice de massa corporal para idosos. Rev Nutr 2005; 18:765-75.

30. Instituto Brasileiro de Geografia e Estatística. Indicadores sociodemográficos e de saúde no Brasil. Rio de Janeiro: Instituto Brasileiro de Geografia e Estatística; 2009. (Estudos e Pesquisas: Informação Demográfica e Socioeconômica, 25).

31. Instituto Brasileiro de Geografia e Estatística. Síntese de indicadores sociais: uma análise das condições de vida da população brasileira. Rio de Janeiro: Instituto Brasileiro de Geografia e Estatística; 2008. (Estudos e Pesquisas: Informação Demográfica e Socioeconômica, 23).

32. Singh-Manoux A, Gueguen A, Martikainen P, Ferrie J, Marmot M, Shipley M. Self-rated health and mortality: short- and long-term associations in Whitehall II study. Psychosom Med 2007; 69:138-43.

33. DeSalvo KB, Jones TM, Peabody J, McDonald J, Fihn S, Fan V, et al. Health care expenditure prediction with a single item, self-rated health measure. Med Care 2009; 47:440-7.

34. Jang Y, Chiriboga DA, Herrera JR, Branch LG. Selfrating of poor health: a comparison of Cuban elders in Havana and Miami. J Cross Cult Gerontol 2009; 24:181-91.

35. McFadden E, Luben R, Bingham S, Wareham N, Kinmonth AL, Khaw KT. Social inequalities in selfrated health by age: cross-sectional study of 22457 middle-aged men and women. BMC Public Health 2008; 8:230.

36. Liang J, Shaw BA, Bennett JM, Krause N, Kobayashi E, Fukaya T, et al. Interwining courses of functional status and subjective health among older Japanese. J Gerontol B Psychol Sci Soc Sci 2007; 62:S340-8.

37. Spencer SM, Schulz R, Rooks RN, Albert SM, Thorpe Jr. RJ, Brenes GA, et al. Racial differences in self-rated health at similar levels os physical functioning: an examination of health pessimism in the health, aging, and body composition study. J Gerontol B Psychol Sci Soc Sci 2009; 64:S87-94.

38. Fonseca MGUP, Firmo JOA, Loyola-Filho AI, Uchôa E. Papel da autonomia na autoavaliação da saúde do idoso. Rev Saúde Pública 2010; 44:159-65.

39. Camarano AA, Kanso S, Leitão e Mello J. Como vive o idoso brasileiro? Rio de Janeiro: Instituto de Pesquisa Econômica Aplicada; 2004.

40. Zaitune MPA, Barros MBA, César CLG, Carandina L, Goldbaum M, Alves MCGP. Fatores associados à prática de atividade física global e de lazer em idosos: Inquérito de Saúde no Estado de São Paulo (ISA-SP), Brasil. Cad Saúde Pública 2010; 26: 1606-18.

41. Lima MG, Barros MBA, César CLG, Carandina L, Goldbaum M, Alves MCGP. Health-related behavior and quality of life among the elderly: a populationbased study. Rev Saúde Pública 2011; 45:485-93.

42. Pearson TA. Alcohol and heart disease. Circulation 1996; 94:3023-5. 
43. American College of Sports Medicine; ChodzkoZajko WJ, Proctor DN, Fiatarone Singh MA, Minson CT, Nigg CR, et al. American College of Sports Medicine position stand. Exercise and physical activity for older adults. Med Sci Sports Exerc 2009; 41:1510-30.

44. Matsudo SM. Envelhecimento, atividade física e saúde. Revista Mineira de Educação Física 2002; 10:195-209.

45. Molarius A, Berglund K, Eriksson C, Lambe M, Nordstrom E, Eriksson HG, et al. Socioeconomic conditions, lifestyle factors, and self-rated health among men and women in Sweden. Eur J Public Health 2006; 17:125-33.
46. Cabrera MAS, Jacob Filho W. Obesidade em idosos: prevalência, distribuição e associação com hábitos e comorbidades. Arq Bras Endocrinol Metab 2001; 45:494-501.

Recebido em 25/Jul/2011

Versão final reapresentada em 31/Out/2011 Aprovado em 21/Nov/2011 